\title{
Combined in-beam gamma-ray and conversion electron spectroscopy with radioactive ion beams
}

\section{Simulations of a silicon detector for the SPEDE spectrometer}

\author{
J. Konki ${ }^{1,2, a}$, P. Papadakis ${ }^{1,2}$, J. Pakarinen ${ }^{1,2}$, P.A. Butler ${ }^{3}$, P. Davies ${ }^{4}$, P.T. Greenlees ${ }^{1,2}$, R.D. Herzberg ${ }^{3}$, M. Huyse ${ }^{5}$, \\ D.G. Jenkins ${ }^{4}$, R. Julin ${ }^{1,2}$, G. O’Neill ${ }^{3}$, R.D. Page ${ }^{3}$, P. Rahkila ${ }^{1,2}$, K. Ranttila1 , J. Thornhill' ${ }^{3}$, and P. Van Duppen ${ }^{5}$ \\ ${ }^{1}$ Department of Physics, University of Jyväskylä, Finland \\ ${ }^{2}$ Helsinki Institute of Physics, Helsinki, Finland \\ ${ }^{3}$ Department of Physics, University of Liverpool, United Kingdom \\ ${ }^{4}$ Department of Physics, University of York, United Kingdom \\ ${ }^{5}$ Instituut voor Kern- en Stralingsfysica, Katholieke Universiteit Leuven, Belgium
}

\begin{abstract}
.
In-beam gamma-ray and electron spectroscopy have been widely used as tools to study the broad variety of phenomena in nuclear structure. The SPEDE spectrometer is a new device to be used in conjunction with the MINIBALL germanium detector array to enable the detection of internal conversion electrons in coincidence with gamma rays from de-exciting nuclei in radioactive ion beam experiments at the upcoming HIE-ISOLDE facility at CERN, Switzerland. Geant 4 simulations were carried out in order to optimise the design and segmentation of the silicon detector to achieve good energy resolution and performance.
\end{abstract}

\section{Introduction and physics motivation}

The structure of the atomic nucleus is a unique and complex quantum mechanical many-body system that has been studied extensively using gamma-ray detector arrays [1,2] and electron spectrometers [3, 4] for decades. However, probing nuclear structure by gamma-ray or electron spectroscopy alone can provide only a limited view of the nuclear de-excitation processes.

Internal conversion competes with gamma-ray emission and in heavy nuclei it can even be the dominant decay mode. Additionally, transitions between low-lying $0^{+}$ states exist frequently in nuclei exhibiting the features of shape co-existence [5], and can only proceed via electric monopole (E0) transitions by emission of an internal conversion electron. In addition, the E0 transition strength can be enhanced for interband $\mathrm{I}^{\pi} \rightarrow \mathrm{I}^{\pi}$ transitions between states of the same spin and parity [6].

Converted transitions play an important role especially in heavy odd-mass nuclei, such as those in the octupole deformed region. There the internal conversion coefficients of the low-energy M1 and E2 transitions are high, which can make the internal conversion the dominant decay mode. Some of these nuclei, namely radon and radium isotopes with $\mathrm{A} \sim 222$, have recently become available as radioactive beams and measurements of octupole deformation employing the Coulex technique for even-even nuclei have been published [7]. However, a complete picture for odd-mass nuclei, important for example for perma-

\footnotetext{
a e-mail: joonas.konki@jyu.fi
}

nent atomic electric-dipole moment studies, can only be achieved via simultaneous electron and gamma-ray spectroscopy. The improved beam intensity, beam quality and higher beam energy from HIE-ISOLDE [8] will make such studies more feasible.

Due to the challenging nature of performing in-beam electron spectroscopy at the target location, relatively few attempts have been made in the past to simultaneously measure in-beam gamma rays and conversion electrons. The best effort up to date is the recently commissioned SAGE spectrometer [9] that is currently used for experiments involving stable ion beams. Moreover, electron spectroscopy has not been exploited to a great extent with intense radioactive ion beams that are provided by a small but growing number of facilities. The SPEDE [10] spectrometer (SPectrometer for Electron DEtection) is a new device that is being constructed and developed to combine internal conversion electron spectroscopy with the existing MINIBALL array [11] used for gamma-ray spectroscopy with radioactive ion beams provided by the upcoming HIEISOLDE facility at CERN.

\section{Experimental setup for SPEDE}

In order to enable simultaneous detection of gamma rays and internal conversion electrons at the target position, the plan is to place the SPEDE silicon detector near the target position of the MINIBALL array. A typical setup for Coulomb excitation experiments with radioactive ion beams has consisted of segmented composite high purity 


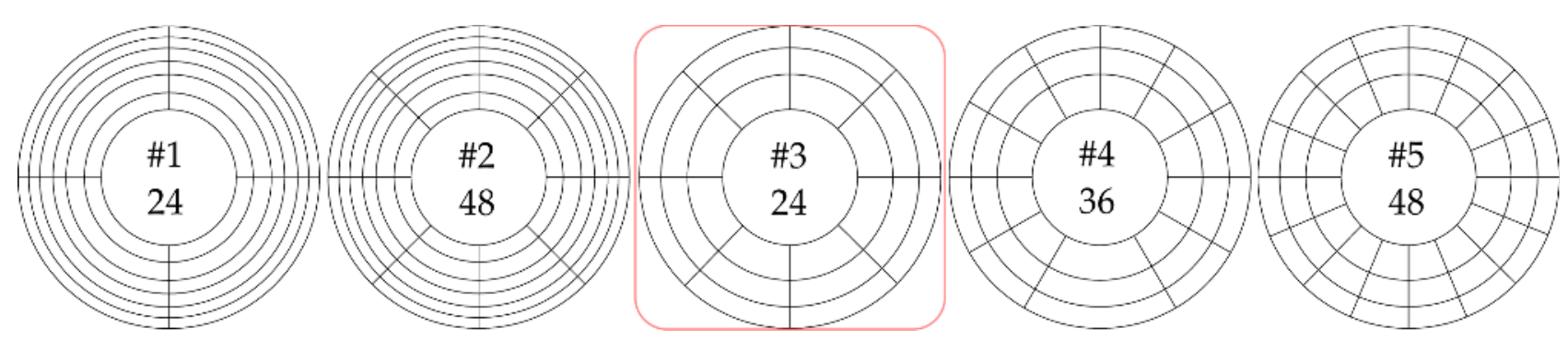

Figure 2. Schematic drawings of the five candidate designs that were simulated for the SPEDE silicon detector. Each design is numbered and the total number of segments are given. Design 3 with 24 segments was chosen for the SPEDE spectrometer.

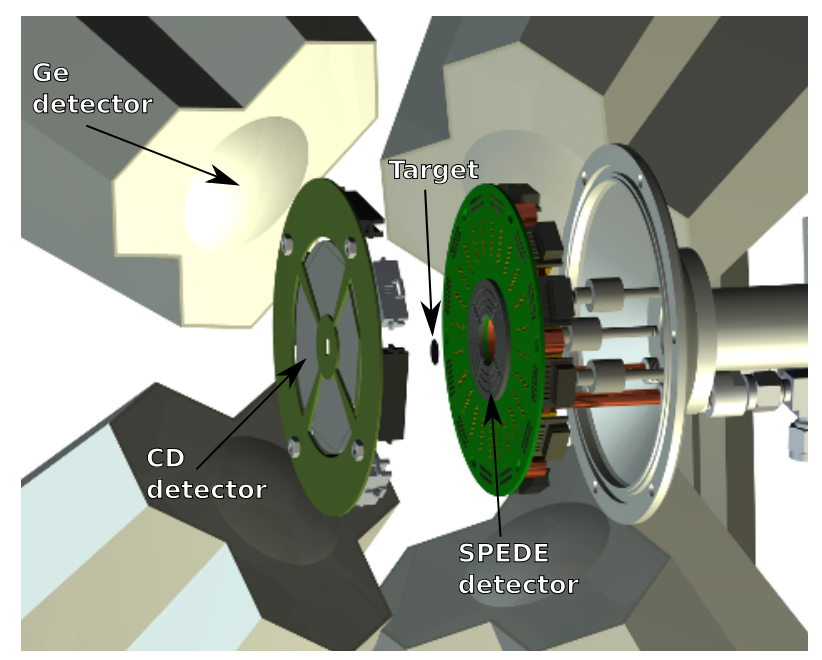

Figure 1. Technical design drawing of the experimental setup used to detect gamma rays and internal conversion electrons simultaneously using the SPEDE spectrometer and the MINIBALL germanium detector array. The radioactive ion beams enter the target chamber from the right and the scattered recoils are detected in the $\mathrm{CD}$ detector placed downstream from the target.

germanium detectors to detect gamma rays emitted at the target position. The recoiling target nuclei or projectile nuclei are detected in a CD shaped double-sided silicon strip detector [12] having 24 radial and 16 annular strips in each of its four quadrants, placed downstream of the target (see figure 1).

The new silicon detector will be placed upstream from the target i.e. at backward angles as shown in Fig. 1. This location is chosen to help reduce background radiation due to e.g. delta-electrons originating from primary beam and target interactions. A target chamber to house the new detector with the existing CD detector is being designed. Furthermore, the geometric segmentation of the silicon detector has to be carefully designed and simulated to achieve reasonable position sensitivity to be able to make kinematic corrections of the measured conversion electron energies (akin to a Doppler correction in the case of gamma rays).

\section{Simulations of a segmented silicon detector for SPEDE}

The Geant4 simulation toolkit $[13,14]$ was used to simulate five candidate geometries with different designs and numbers of segments for the silicon detector of the SPEDE spectrometer. The designs that were considered are shown in Fig. 2. The CD detector was included in the simulation geometry to enable calculation of the kinematic correction. An illustration of the complete simulation geometry in Geant 4 for one of the detector designs is shown in Fig. 3. A $1000 \mu \mathrm{m}$ thick silicon detector was placed $25 \mathrm{~mm}$ upstream from the target and the CD detector $33 \mathrm{~mm}$ downstream from the target. The green areas represent the printed circuit boards on which the silicon wafers are mounted.
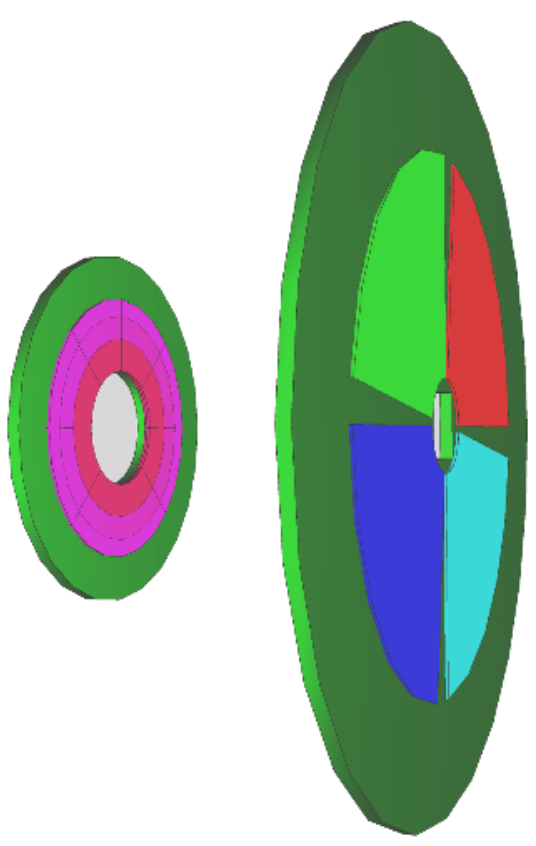

Figure 3. Schematic representation of the simulated geometry in Geant4 including the SPEDE detector design 3 (left) and the $\mathrm{CD}$ detector (right). Beam direction is from left to right. The surrounding sections in green illustrate the printed circuit board material on which the silicon wafers are mounted. The other colours represent silicon in the quadrants of the $\mathrm{CD}$ detector and the SPEDE detector 
A test case in the simulations was Coulomb excitation of a ${ }^{188} \mathrm{~Pb}$ beam impinging on a $2 \mathrm{mg} / \mathrm{cm}^{2}$ thick ${ }^{112} \mathrm{Cd}$ target at an energy of $2.82 \mathrm{MeV} / \mathrm{u}$. The beam spot size was assumed to be Gaussian with width of $3 \mathrm{~mm}$ (FWHM). The reaction was set to take place at the centre of the target, populating the proposed excited $0_{3}^{+}$state at $767 \mathrm{keV}$ via the first excited $2^{+}$state. The differential cross section distribution populating this state was approximated to be similar to an E2 transition as presented in Fig. 4 for the scattered, excited projectile-like particles in the centre of mass frame of reference. This distribution was used to create the primary events in the simulation. For simplicity, Rutherford scattering and the recoiling ${ }^{112} \mathrm{Cd}$ target nuclei were left out of this study.

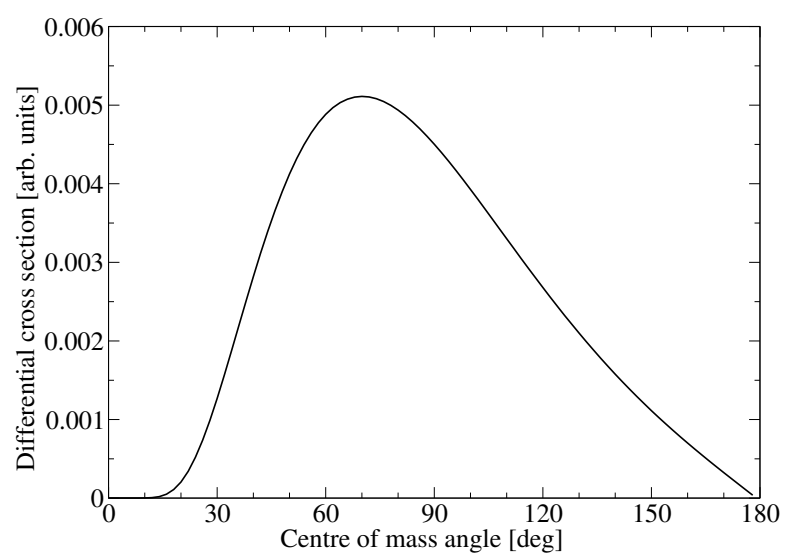

Figure 4. Angular distribution in the centre of mass frame of reference of the scattered and excited ${ }^{188} \mathrm{~Pb}$ particles that was used as input in the simulations.

The resulting reaction kinematics in the laboratory frame of reference that were calculated and used as input in the simulation is illustrated in Fig. 5. The CD detector does not cover all the angles that the projectiles are scattered into, and these events were excluded from the simulation. An intrinsic detector resolution of $3 \mathrm{keV}$ was assumed for all of the designs by applying a Gaussian distribution to the electron energies deposited in the silicon detector. The projectile energy loss in the target material, the SPEDE silicon detector dead layers and strip gaps were not incorporated in the simulations.

\section{Simulation results}

For each detector design candidate, one million events were simulated where a recoil projectile emits the Kconversion electron of energy $678 \mathrm{keV}$ from the $767 \mathrm{keV}$ transition. The simulation results were analysed using the GRAIN data analysis package [15]. As an example of the effect of the kinematic correction applied to the simulated transition, the electron energy spectrum obtained from the silicon detector design 3 is shown in Fig. 6 where recoilgated electron spectra are shown with and without the correction. The improvement in the peak shape when applying the correction is evident and the energy resolution is

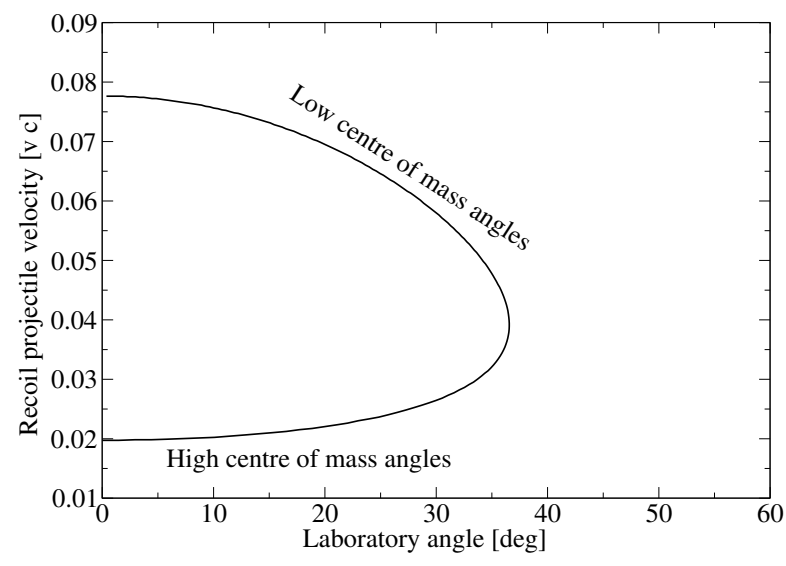

Figure 5. Simulated event kinematics considered only scattered projectile-like particles of ${ }^{188} \mathrm{~Pb}$. The two different kinematic branches resulting from inverse kinematics have been labelled in the plot.

comparable to the performance of the MINIBALL germanium detector array.

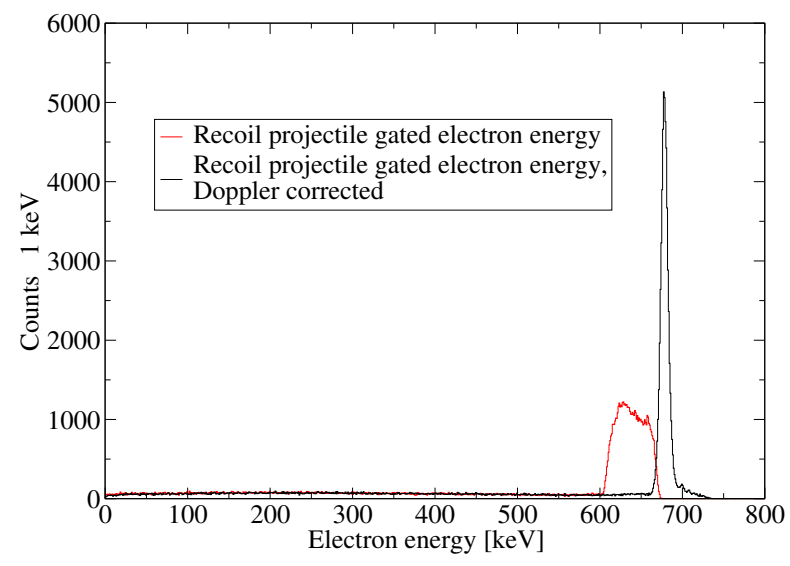

Figure 6. Recoil-gated uncorrected (red) and kinematically corrected (black) electron energy spectra for the chosen detector design 3. The K-conversion line of the $767 \mathrm{keV} \mathrm{E0}$ transition in ${ }^{188} \mathrm{~Pb}$ is recovered by using the low centre of mass kinematics for the correction.

In contrast to a real measurement, the kinematic correction in the simulation is more ideal and simplified in that it uses a value for the recoil velocity obtained from the calculated velocities in the low centre of mass angle branch of Fig. 5 combined with the known angle of the $\mathrm{CD}$ detector strip. In reality, the recoil velocity is determined from the energy deposited in the $\mathrm{CD}$ where the energy resolution is worse and cannot always be distinguished from target-like recoils.

A comparison of the detector designs in terms of spectral response when the kinematic correction is applied is shown in Fig. 7. The key parameters for each design are summarised in Table 1 where the energy resolutions are given for the $678 \mathrm{keV}$ peak (FWHM) as well as the average segment area. 
Table 1. Key parameters and results obtained from the Geant 4 simulations for the different silicon detector designs in Fig. 2.

\begin{tabular}{cccc}
\hline Design & Segments & $\begin{array}{c}\text { FWHM @ } \\
678 \mathrm{keV}[\mathrm{keV}]\end{array}$ & $\begin{array}{c}\text { Avg. segment } \\
\text { area }\left[\mathrm{mm}^{2}\right]\end{array}$ \\
\hline 1 & 24 & 12.6 & 53.2 \\
2 & 48 & 9.0 & 26.6 \\
3 & 24 & 9.2 & 53.2 \\
4 & 36 & 8.6 & 39.9 \\
5 & 48 & 8.1 & 26.6 \\
\hline
\end{tabular}

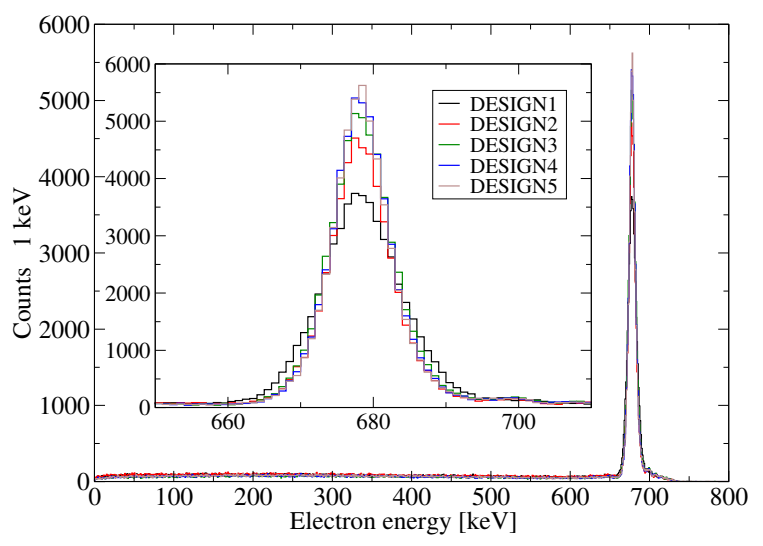

Figure 7. Recoil gated and kinematically corrected electron energy spectra for the K-conversion line of the $767 \mathrm{keV}$ E0 transition in ${ }^{188} \mathrm{~Pb}$ from all five detector designs from the simulation. See text for more details.

As a result of the simulations, design 3 was chosen for the SPEDE detector. The larger capacitance and leakage current for the design with fewer strips will increase the intrinsic resolution, however, this may not be noticeable if the overall resolution will be about $9 \mathrm{keV}$ due to the kinematic broadening. Detectors with a thickness of $500 \mu \mathrm{m}$ have been purchased from Micron Semiconductor Ltd. A photograph of one of the detectors without the printed circuit board is shown in Fig. 8.

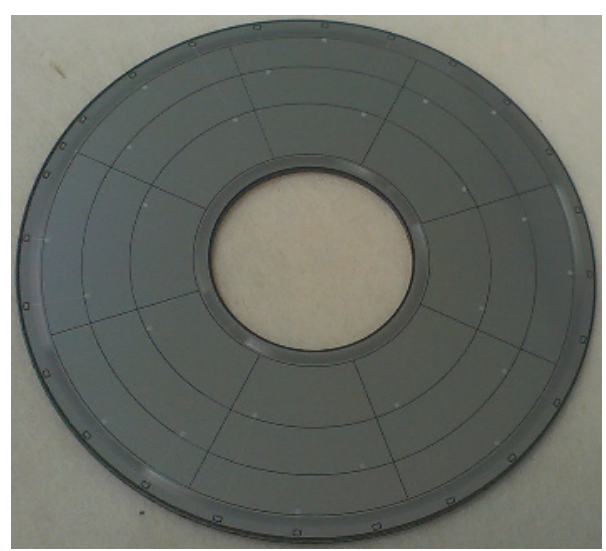

Figure 8. Photograph of the purchased SPEDE silicon detector. The detector is $500 \mu \mathrm{m}$ thick and has 24 segments.

\section{Conclusions}

It can be concluded from Fig. 7 and Table 1, that instead of only four annular segments, dividing the detector rings annularly in eight or more yields better performance. On the other hand, 16 annular segments per ring (design 5) would not make significant improvement in resolution compared to twelve (design 4) or eight (design 3). The same applies for the radial direction, where higher segmentation does not boost performance remarkably when comparing design 2 with design 3 . Taking into account the number of electronics channels needed to accommodate the detector and achievable gain in the final kinematically corrected spectra, the design 3 with 24 segments is the preferred choice.

\section{Acknowledgements}

Funding is gratefully acknowledged from the United Kingdom Science and Technology Facilities Council (UK STFC). This work is supported by the Marie Curie Career Integration Grant (Grant No. 304033) and by the Academy of Finland (Grant No. 257562).

\section{References}

[1] C.W. Beausang, J. Simpson, Journal of Physics G: Nuclear and Particle Physics 22, 527 (1996)

[2] J. Eberth, J. Simpson, Progress in Particle and Nuclear Physics 60, 283 (2008)

[3] P.A. Butler et al., Nuclear Instruments and Methods in Physics Research A 381, 433 (1996)

[4] M. Metlay et al., Nuclear Instruments and Methods in Physics Research Section A 336, 162 (1993)

[5] K. Heyde, J.L. Wood, Rev. Mod. Phys. 83, 1655 (2011)

[6] C. Wu et al., Physics Letters B 541, 59 (2002)

[7] L.P. Gaffney et al., Nature 497, 199 (2013)

[8] M. Lindroos et al., Nuclear Instruments and Methods in Physics Research B 266, 4687 (2008)

[9] P. Papadakis et al., AIP Conference Proceedings 1090, 14 (2009)

[10] J. Pakarinen et al., Letter of intent to the INTC for HIE-ISOLDE (2010)

[11] J. Eberth et al., Progress in Particle and Nuclear Physics 46, 389 (2001)

[12] A. Ostrowski et al., Nuclear Instruments and Methods in Physics Research A 480, 448 (2002)

[13] S. Agostinelli et al., Nuclear Instruments and Methods in Physics Research A 506, 250 (2003)

[14] J. Allison et al., IEEE Transactions on Nuclear Science 53, 270 (2006)

[15] P. Rahkila, Nuclear Instruments and Methods in Physics Research A 595, 637 (2008) 